Research Article

\title{
Phylogenomic and single nucleotide polymorphism analyses revealed the hybrid origin of Spondias bahiensis (family Anacardiaceae): de novo genome sequencing and comparative genomics
}

\author{
Lydayanne Lilás de Melo Nobre ${ }^{1}$, José Daniel Oliveira dos Santos ${ }^{1}$, Rychard Leite ${ }^{1}$ and Cícero Almeida ${ }^{1}$ (D) \\ ${ }^{\text {I} G e n e t i c ~ R e s o u r c e s ~ L a b o r a t o r y, ~ A r a p i r a c a ~ C a m p u s, ~ U n i v e r s i d a d e ~ F e d e r a l ~ d e ~ A l a g o a s, ~ A r a p i r a c a, ~ A L, ~}$ \\ Brazil.
}

\begin{abstract}
The genus Spondias (family Anacardiaceae) comprises 19 taxa, ten of which occur in Neotropical regions. Spondias bahiensis has been suggested to be a hybrid, although initial evidence does not support this hypothesis. The aim of this study was to test the hypothesis of the hybrid origin of $S$. bahiensis using high-throughput sequencing with single nucleotide polymorphism (SNP) analysis, characterization of intragenomic nuclear ribosomal DNA (nrDNA), and nuclear and chloroplast phylogenomic analyses. The SNP analysis revealed a high number of SNPs in the S. bahiensis genome, and with respect to nrDNA, S. bahiensis shared approximately half of the SNP alleles with $S$. tuberosa, but not with $S$. mombin. Combining the SNP analysis with nrDNA phylogeny confirmed the hybrid origin of $S$. bahiensis and put $S$. tuberosa as the female genitor. Considering the phylogeny of the genus Spondias and intraspecific SNPs in $S$. bahiensis, the putative male genitor is $S$. dulcis.
\end{abstract}

Keywords: rDNA, phylogenetics, evolution, hybrid offspring.

Received: August 13, 2017; Accepted: February 23, 2018.

\section{Introduction}

The genus Spondias (family Anacardiaceae) comprises 19 taxa, 10 of which occur in Neotropical regions (Mitchell and Daly, 2015). Spondias tuberosa Arruda, S. mombin L., and S. purpurea L. are widespread most notably in northeastern Brazil, in addition to a new species (Spondias bahiensis P. Carvalho, van den Berg \& M. Machado), which was recently described by Machado et al. (2015). Spondias tuberosa, S. mombin and S. bahiensis, which are known by the vernacular names umbu, cajá, taperebá, and umbu-cajá, occur in northeastern Brazil. Spondias bahiensis has been popularly known as a hybrid between S. tuberosa and S. mombin, but this hypothesis was contested by chromosome banding and genomic in situ hybridization (GISH) studies (Almeida et al., 2007), DNA barcoding (Silva et al., 2015), and molecular and morphological analyses (Machado et al., 2015). Machado et al. (2015) discussed the possibility that $S$. bahiensis could have originated by hybridization between $S$. tuberosa and $S$. venulosa, but there is no evidence in support of this hypothesis either. In this context, a recent study used high-

Send correspondence to Cicero de Almeida. Arapiraca Campus, Universidade Federal de Alagoas, Avenida Manoel Severino Barbosa s/n, Rodovia AL 115, km 6,5. Bairro Bom Sucesso, 57309-005 Arapiraca, AL, Brazil. E-mail: cicero@ arapiraca.ufal.br throughput sequencing data to investigate the origin of $S$. bahiensis and the evolution of the genus Spondias.

Molecular phylogenetic analysis is essential to elucidate the ecological, evolutionary, and taxonomic characteristics of organisms. To date, most phylogenetic methods have used only a tiny portion of the nuclear and chloroplast genomes, generally the phylogeny of genes or intergenic spacers. With next generation sequencing technologies, whole genome sequence data have become faster and cheaper to obtain, and phylogenetic reconstruction has dramatically accelerated, for instance, high-throughput sequencing has facilitated phylogenomic studies based on multilocus phylogeny (Comer et al., 2015), repetitive DNA (satellites and mobile elements) (Macas et al., 2015), chloroplast genomic DNA (Barrett et al., 2016), and nuclear ribosomal DNA (nrDNA) (Weitemier et al., 2015). Although nrDNA is not suitable for phylogenetic studies because the copies are assembled as tandem repeats and homologous recombination occurs frequently, nrDNA alleles may become species-specific, thereby allowing hybrid detection. Single nucleotide polymorphism (SNP) analysis has been utilized in studies of genomic characterization and diversity, but heretofore the SNP approach has not been used for genomic analysis of hybrids. The aim of the present study was to determine the hybrid origin of $S$. bahiensis using highthroughput sequencing to analyze SNP variants, nrDNA alleles, and the nuclear and chloroplast phylogenomics. 


\section{Material and Methods}

\section{High-throughput sequence data}

Samples of S. tuberosa, S. mombin, and S. bahiensis were collected in the state of Alagoas, Brazil, and DNA extraction was performed from leaves using the cetyl trimethylammonium bromide extraction method, as described by Doyle and Doyle (1987). The quality and quantity of the extracted DNA were verified by visualization on a $1 \%$ agarose gel and spectrophotometry, respectively. Species identification was performed using the DNA barcodes described by Silva et al. (2015). DNA samples were fragmented into 400-500 bp to construct a sequencing library. The fragments were ligated with adapters using the Nextera DNA Sample Preparation kit (Illumina, Inc., San Diego, CA, USA). Sequencing of 100-nt single-end reads for $S$. bahiensis and 100-nt paired-end reads for S. tuberosa and S. mombin was performed using the Illumina HiSeq2500 platform at the Central Laboratory for High Performance Technologies in Life Sciences (LaCTAD) of the State University of Campinas (São Paulo, Brazil). For Pistacia vera (utilized as outgroup), sequence read archive (SRA) files were unpacked into FASTQ using the FASTQ-DUMP executable function from the SRA toolkit. FASTQ files were then filtered with a minimum quality of 10 and converted to FASTA files.

\section{Phylogenomic analysis}

Chloroplast genome sequences were obtained from the National Center for Biotechnology Information database (https://www.ncbi.nlm.nih.gov/) and aligned using the MAFFT v7.017 algorithm (Katoh and Standley, 2013) implemented as the "Multiple align" tool in Geneious R9 (http://www.geneious.com). Bayesian analysis was performed using Beast v2 (Drummond and Rambaut, 2007) and posterior distribution was approximated using the Markov chain Monte Carlo (MCMC) method with 10 million steps. Convergence of the parameters was checked using the Tracer 1.5 program (Rambaut et al., 2018). For nuclear phylogenomic analysis, the phylogenies of the reads were reconstructed using the assembly and alignment-free (AAF) method (Fan et al., 2015), and phylogenetic relationships were estimated using high-throughput sequencing data from the whole genomes. The phylogenies were reconstructed directly from unassembled genome sequence data.

\section{Discovery and analysis of SNPs}

For SNP analysis, the de novo contigs were constructed from 198 million reads from S. tuberosa using Ray software (Boisvert et al., 2012), with a minimum size of $200 \mathrm{nt}, 31 \mathrm{k}-\mathrm{mers}$, and 8 coverage. The largest contigs were then used as references. The data from $S$. tuberosa, $S$. bahiensis, and $S$. mombin were aligned for SNP identification using GATK software (Van der Auwera et al., 2013).
The results, in vcf format, were analyzed using $\mathrm{R}$ package (vcfR packager) (Knaus and Grunwald, 2017).

\section{Nuclear ribosomal analysis}

The paired-end reads from $S$. mombin were utilized for repeat analysis, which was performed with Tandem Repeat Analyzer (TAREAN) software (Novák et al., 2017) for repeat identification. TAREAN is a computational pipeline for identification of repeats from unassembled sequence reads. After identification of the nrDNA, the spacer regions ITS1 and ITS2 were utilized for allele identification by sequence mapping. A phylogenetic tree of the alleles was constructed for characterization of intragenomic nrDNA polymorphisms. The sequences were aligned using the MAFFT v7.017 program (Katoh and Standley, 2013) implemented as the "Multiple align" tool in Geneious R9 (http://www.geneious.com). Bayesian analysis was performed using Beast v2 (Drummond and Rambaut, 2007) and posterior distribution was approximated using the MCMC method with 10 million steps. The convergence of the parameters was checked using the Tracer 1.5 program (Rambaut et al., 2018). Cluster analysis by similarity-based clustering of Illumina reads was performed using RepeatExplorer (Novák et al., 2010) individually for ITS1 and ITS2.

\section{Results}

\section{Phylogenomic relationships}

The phylogenomic analysis using complete chloroplast genomes (Figure 1A) and nuclear data obtained by the AAF approach (Figure 1B) revealed three clades: one formed by $S$. tuberosa and $S$. bahiensis, an intermediary clade consisting of $S$. mombin, and an out-group clade formed by Pistacia vera and Rhus chinensis for the chloroplast tree and Pistacia vera for the nuclear phylogenomic tree. Pairwise comparisons for chloroplast analyses identified 856 SNPs between $S$. tuberosa and S. bahiensis, 3042 between S. tuberosa and S. mombin, and 3292 between S. bahiensis and S. mombin.

\section{SNP analysis}

For SNP discovery, six contigs, ranging in length from 48,859 to $54,663 \mathrm{bp}$, were analyzed with GATK software. The results revealed 0-31 SNPs in S. tuberosa and 0-48 in S. mombin, with a mean of two and five SNPs for each $10 \mathrm{~kb}$ (Table 1 and Figure 2). Remarkably, $S$. bahiensis showed the highest SNP content of 678-936 SNPs, with a mean of 166 SNPs for each $10 \mathrm{~kb}$. The detailed analysis for contig F showed that $S$. bahiensis shared only half of the alleles with $S$. tuberosa, indicating that $S$. tuberosa is a genitor of $S$. bahiensis (Figure 3). 


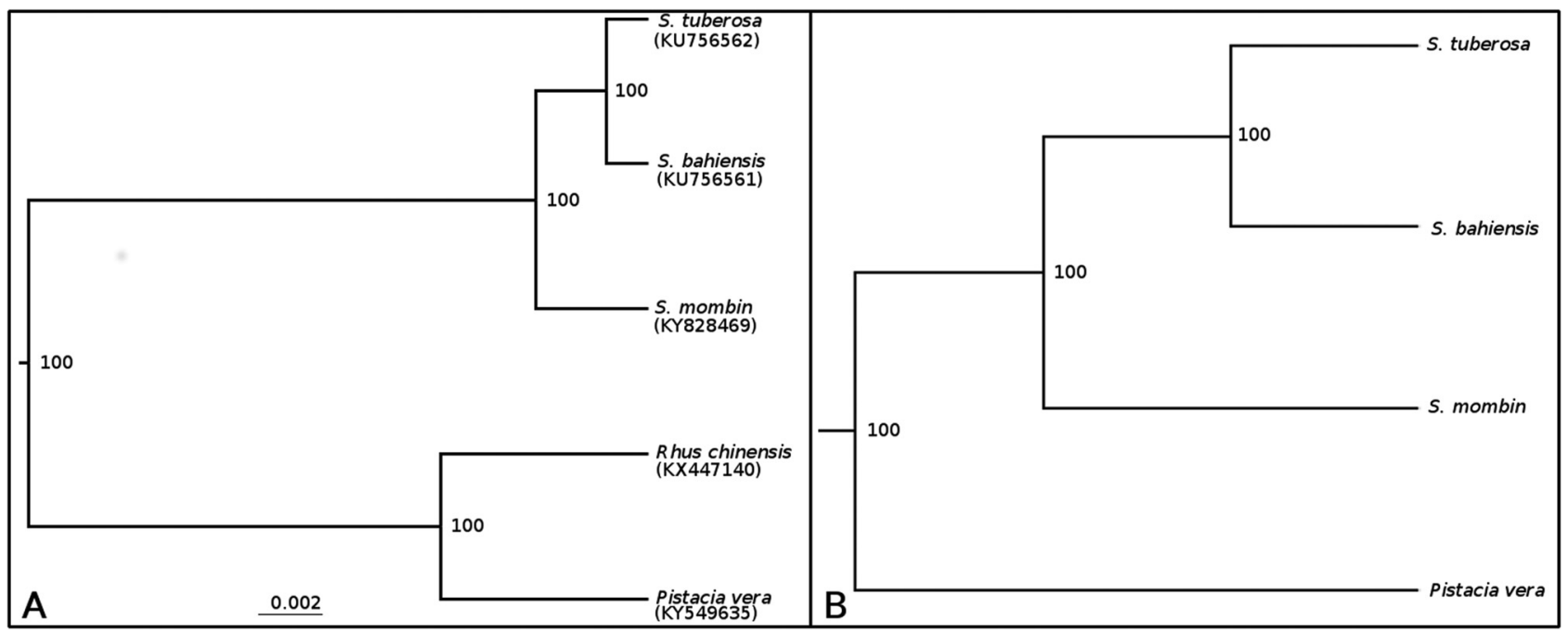

Figure 1 - Phylogenetic analysis of the chloroplast and nuclear genomes. (A) Phylogenetic relationships using the Bayesian approach of the complete chloroplast genomes of the genus Spondias and out group Rhus chinenesis and Pistacia vera. The support values are estimated with posterior probabilities (in percentages). (B) A phylogenetic tree reconstructed with the AAF approach using nuclear reads. The support values are estimated with bootstrap values (in percentages).

Table 1 - Number of SNPs present in six contigs for Spondias tuberosa, Spondias bahiensis, and Spondias mombin.

\begin{tabular}{lccccc}
\hline & & \multicolumn{3}{c}{ Intra-specific } & \\
\cline { 3 - 5 } Contig & $\begin{array}{c}\text { Length } \\
\text { (bp) }\end{array}$ & $\begin{array}{c}S . \\
\text { tuberosa }\end{array}$ & $\begin{array}{c}S . \\
\text { bahiensis }\end{array}$ & $\begin{array}{c}\text { S. } \\
\text { mombin }\end{array}$ & $\begin{array}{c}\text { Inter- } \\
\text { specific* }\end{array}$ \\
\hline Contig A & 54,663 & 24 & 936 & 39 & 946 \\
Contig B & 51,123 & 6 & 892 & 0 & 621 \\
Contig C & 49,173 & 0 & 838 & 13 & 778 \\
Contig D & 47,249 & 1 & 828 & 18 & 676 \\
Contig E & 50,619 & 1 & 834 & 48 & 821 \\
Contig F & 48,859 & 31 & 678 & 30 & 835 \\
Total & 301,686 & 66 & 5,006 & 148 & 4,677 \\
\hline
\end{tabular}

* Variation between S. tuberosa and S. mombin.

\section{Nuclear ribosomal phylogeny}

The allele identification for ITS1 and ITS2 of Spondias and Pistacia revealed five alleles for ITS1 (H1-H5) (Figure 4A) and eight for ITS2 (H1-H8) (Figure 4C). Among the ITS1 alleles, H1 was exclusive to $P$. vera (outgroup), $\mathrm{H} 2$ and $\mathrm{H} 3$ to $S$. mombin, $\mathrm{H} 4$ to $S$. bahiensis, and H5 to both S. tuberosa and S. bahiensis, indicating that $S$. tuberosa and $S$. bahieneis shared the same alleles. The intraspecific variation observed between $\mathrm{H} 2$ and $\mathrm{H} 3$ for ITS1 (S. mombin) was limited to only one SNP.

For ITS2, the phylogenetic relationships showed the same topology. S. tuberosa and S. bahiensis showed a high relationship with allele differences of only one SNP (alleles H6 and H7). Intraspecific variation was observed in $S$. tuberosa and S. mombin, with one and two SNPs, respectively. Similar to ITS1, ITS2 analyses also suggested that $S$. tuberosa is the genitor of $S$. bahiensis. Graph analysis of ITS1 (Figure 4B) and ITS2 (Figure 4D) using reads from both species showed clustering of dots corresponding to the specific species. Notably, reads from $S$. tuberosa and $S$. bahiensis were overlapping, while reads from $S$. mombin were not overlapping, suggesting that $S$. tuberosa and $S$. bahiensis are closely related.

\section{Discussion}

The phylogenetic relationships in the genus Spondias have been supported by molecular data (Silva et al., 2015; Machado et al., 2015) and cytogenetic data (Almeida et al., 2007). These studies discuss a possible hybrid origin of a plant popularly known "umbu-cajá" from $S$. tuberosa and S. mombin. This was not supported by chromosome banding or GISH studies (Almeida et al., 2007). Furthermore, phylogenetic studies of chloroplast regions and expressed sequence tags (Machado et al., 2015; Silva et al., 2015) suggested that umbu-cajá is a distinct species, which was named $S$. bahiensis by Machado et al. (2015).

Here, a combination of different approaches was utilized to assess the hybrid origin of $S$. bahiensis. The approaches included the identification and analysis of the ITS1 and ITS2 alleles, phylogenomic analysis of the complete chloroplast and nuclear genomes, and exhaustive SNP analysis. This is the first study to use nrDNA alleles and SNP approaches to identify the genomic sequence of a hybrid. The results of this study demonstrated the validity of these approaches. Usually, GISH is utilized to distinguish cellular genomes and it has been an important tool for molecular cytogenetics (Silva and Souza, 2013; Ramzan et al., 2017). However, GISH of the genus Spondias, using labeled total DNA of S. mombin or S. tuberosa as a probe and hybridized on metaphase chromosomes of S. bahiensis, revealed similar results between the probes, suggesting that there was high identity between the genomes. In this con- 


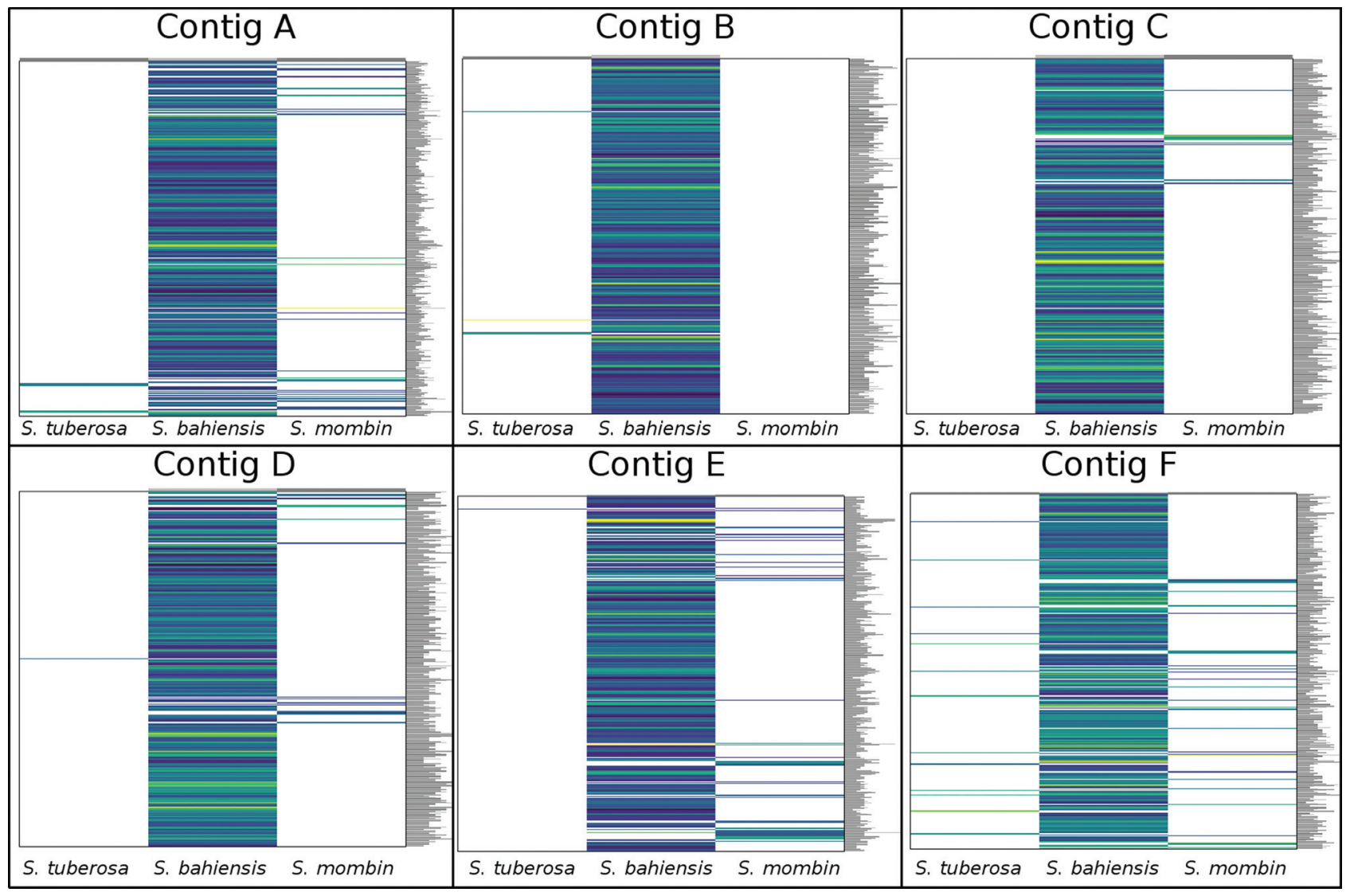

Figure 2 - SNP distribution and density of six contigs in Spondias tuberosa, Spondias bahiensis, and Spondias mombin.

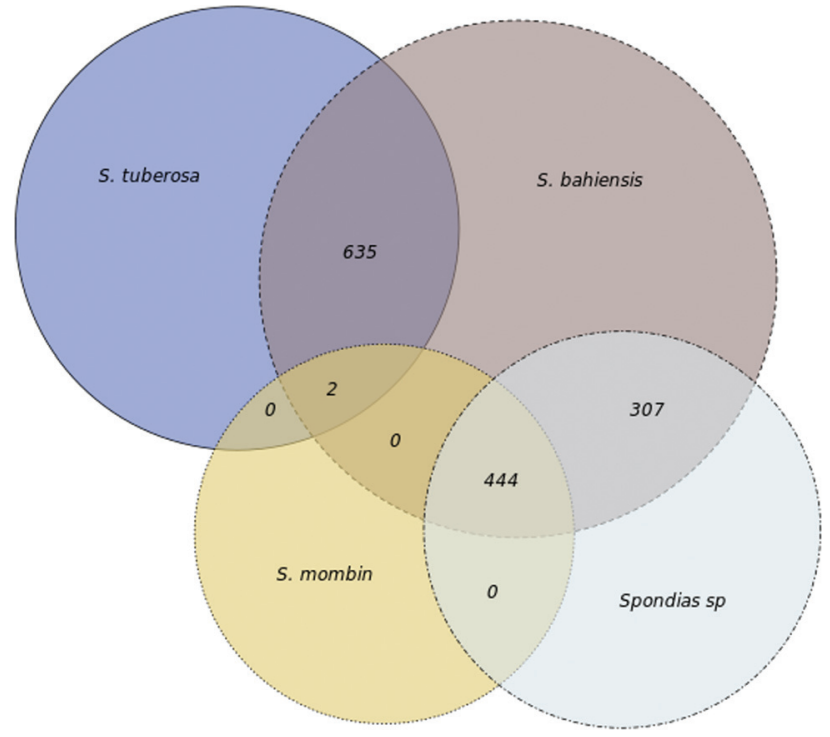

Figure 3 - Venn diagram of the allelic distribution of Spondias bahiensis. The Venn diagram shows the number of alleles that are shared among Spondias bahiensis, Spondias tuberosa, and Spondias mombin.

text, the present study showed that SNP analysis is effective for detecting differences in the genomes of naturally occurring hybrids.
The phylogenomic analysis results corroborated the findings of previous studies (Machado et al., 2015; Silva et al., 2015) that showed $S$. bahiensis as genetically intermediate between $S$. tuberosa and $S$. mombin, suggesting a hybrid origin. Pairwise comparisons of the chloroplast genomes showed high identity between $S$. tuberosa and $S$. bahiensis, indicating $S$. tuberosa as the female genitor of the putative hybrid $S$. bahiensis (in Anacardiaceae, the chloroplast DNA is inherited only from the female genitor). The hybrid origin of $S$. bahiensis was confirmed by SNP analysis, which revealed a high number of SNPs in the contigs, with 75.8 and 33.8 more SNPs for S. bahiensis than for $S$. tuberosa and $S$. mombin, respectively. Analysis of the ITS alleles showed that $S$. bahiensis shared identical or nearly identical alleles with $S$. tuberosa, suggesting $S$. tuberosa as a genitor. The results furthermore indicate that $S$. mombin is not a genitor, as $S$. mombin did not share alleles with $S$. bahiensis. These conclusions are in agreement with the findings of the SNP analyses, showing that half of the alleles of $S$. bahiensis originated from $S$. tuberosa, while the other half were not exclusive to $S$. mombin, suggesting that $S$. mombin is not a genitor of $S$. bahiensis. Because the fruits of $S$. tuberosa, S. mombin, and S. bahiensis are consumed in Brazil, this conclusion has practical implications, as it helps to stabilize the germplasm banks and breeding strategies for these three species. Notably, the ge- 


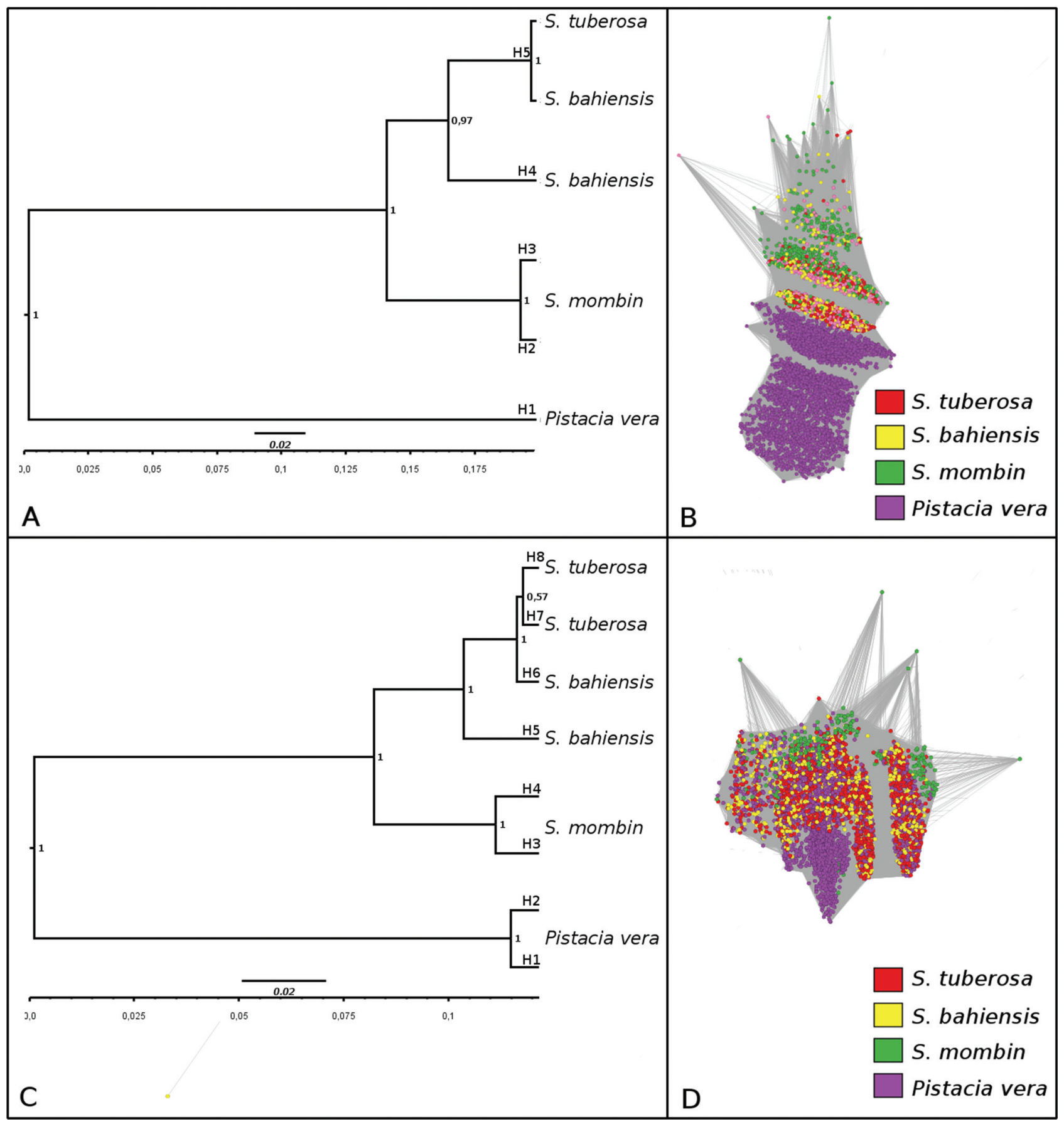

Figure 4 - Phylogenetic analysis of ITS1 and ITS2. Phylogenetic relationships between ITS1 (A) and ITS2 (C) using the Bayesian approach. Graphic representation of ITS1 (B) and ITS2 (D), where reads from the species are highlighted in red (Spondias tuberosa), yellow (Spondias bahiensis), green (Spondias mombin), and purple (Pistacia vera).

nus Spondias is propagated by seeds and vegetative means (Espíndola et al., 2004) while S. bahiensis must be propagated vegetatively, as the hybrid origin leads to segregation among progenies.

\section{References}

Almeida CC, De Lemos Carvalho PC and Guerra M (2007) Karyotype differentiation among Spondias species and the putative hybrid Umbu-cajá (Anacardiaceae). Bot J Linn Soc 155:541-547.

Barrett CF, Baker WJ, Comer JR, Conran JG, Lahmeyer SC, Leebens-Mack JH, Li J, Lim GS, Mayfield-Jones DR, Perez L et al. (2016) Plastid genomes reveal support for deep phylogenetic relationships and extensive rate variation among palms and other commelinid monocots. New Phytol 209:855-870. 
Boisvert S, Raymond F, Godzaridis E, Laviolette F and Corbeil J (2012) Ray Meta: Scalable de novo metagenome assembly and profiling. Genome Biol 13:R122.

Comer JR, Zomlefer WB, Barrett CF, Davis JI, Stevenson DW, Heyduk K and Leebens-Mack JH (2015) Resolving relationships within the palm subfamily Arecoideae (Arecaceae) using plastid sequences derived from next-generation sequencing. Am J Bot 102:888-899.

Doyle JJ and Doyle JL (1987) A rapid DNA isolation procedure for small quantities of fresh leaf tissue. Phytochem Bulletin 19:11-15.

Drummond AJ and Rambaut A (2007) BEAST: Bayesian evolutionary analysis by sampling trees. BMC Evolutionary Biology 7:214.

Espíndola ACM, Almeida CCS, Carvalho NSG and Roza ML (2004) Diâmetro do caule e método de enxertia na formação de mudas de umbuzeiro (Spondias tuberosa Arruda Cam). Curr Agric Sci Technol 10:371-372.

Fan H, Ives AR, Surget-Groba Y and Cannon CH (2015) An assembly and alignment-free method of phylogeny reconstruction from next-generation sequencing data. BMC Genomics 16:522.

Katoh K and Standley DM (2013) MAFFT multiple sequence alignment software version 7: Improvements in performance and usability. Mol Biol Evol 30:772-780.

Knaus BJ and Grunwald NJ (2017) vcfr: A package to manipulate and visualize variant call format data in R. Mol Ecol Resour $17: 44-53$.

Macas J, Novak P, Pellicer J, Cizkova J, Koblizkova A, Neumann P, Fukova I, Dolezel J, Kelly LJ and Leitch IJ (2015) In depth characterization of repetitive DNA in 23 plant genomes reveals sources of genome size variation in the legume tribe Fabeae. PLoS One 10:e0143424.

Machado M, Carvalho P and van den Berg C (2015) Domestication, hybridization, speciation, and the origins of an eco- nomically important tree crop of Spondias (Anacardiaceae) from the Brazilian Caatinga dry forest. Neodiversity 8:8-49.

Mitchell JD and Daly DC (2015) A revision of Spondias (Anacardiaceae) in the Neotropics. Phytokeys 55:1-92.

Novák P, Ávila Robledillo L, Koblíková A, Vrbová I, Neumann P and Macas J (2017) TAREAN: A computational tool for identification and characterization of satellite DNA from unassembled short reads. Nucleic Acids Res 45:e111.

Novák P, Neumann P and Maca J (2010) Graph-based clustering and characterization of repetitive sequences in next-generation sequencing data. BMC Bioinformatics 11:378.

Rambaut A, Drummond AJ, Xie D, Baele G and Suchard MA (2018) Posterior summarisation in Bayesian phylogenetics using Tracer 1.7. Syst Biol 67:901-904.

Ramzan F, Younis A and Ki-Byung Lim (2017) Application of genomic in situ hybridization in horticultural science. Int $\mathrm{J}$ Genomics 2017:7561909.

Silva GS and Souza MM (2013) Genomic in situ hybridization in plants Genet Mol Res 12:2953-65.

Silva JN, Costa A, Silva JV and Almeida C (2015) DNA barcoding and phylogeny in Neotropical species of the genus Spondias. Biochem Syst Ecol 61:240-243.

Van der Auwera GA, Carneiro M, Hartl C, Poplin R, del Angel G, Levy-Moonshine A, Jordan T, Shakir K, Roazen D, Thibault J et al. (2013) From FastQ data to high-confidence variant calls: The Genome Analysis Toolkit best practices pipeline. Curr Protoc Bioinformatics 43: 11.10.1-33.

Weitemier K, Straub SCK, Fishbein M and Liston A (2015) Intragenomic polymorphisms among high-copy loci: A genus-wide study of nuclear ribosomal DNA in Asclepias (Apocynaceae). PeerJ 3:e718.

\section{Associate Editor: Everaldo Gonçalves de Barros}

License information: This is an open-access article distributed under the terms of the Creative Commons Attribution License (type CC-BY), which permits unrestricted use, distribution and reproduction in any medium, provided the original article is properly cited. 